\title{
Review on Various Routing Attacks in Vehicular Adhoc Networks
}

\author{
Jayant Vasu \\ Research Scholar, \\ Shri Venkateshwara University, \\ Gujraula, India
}

\author{
Gaurav Tejpal, PhD \\ Professor, \\ Shri Venkateshwara University, \\ Gujraula, India
}

\author{
Sonal Sharma, $\mathrm{PhD}$ \\ Assistant Professor, \\ Department of Computer \\ Applications, Uttaranchal University \\ Dehradun, India
}

\begin{abstract}
Vehicular adhoc networks (VANETS) really are an stimulating technology which innovates to allow the communication among vehicles utilizing side as well as among cars with street area devices on the other side. VANETS provide a large quantity of programs without the help from repaired infrastructure. These programs ahead communicate in a multi-hop fashion. Planning an effective routing method for several VANET programs is extremely hard. Security is an essential matter for routing in VANETS, since various applications will effect life-or-death decisions as well as illegal tampering could have disturbing consequences. The overall objective of this paper is to explore the various routing attacks in VANETs.
\end{abstract}

\section{Keywords}

VANETS, Taxonomy of Routing Protocols, Attacks in VANETS

\section{INTRODUCTION}

Internetworking in VANETS has been attaining lots of strength in the last number of years. Its rising value has been identified by big car makers, governmental corporations, as well as the educational community [8]. VANETS might actually build to become ideal for road journey defense in addition to many industrial applications [1]. For instance, vehicular network could possibly build to become ideal for road path safety along with many industrial applications [1]. For example, vehicular system can be utilized to know further about the visitor's jams, giving larger ease along with performance [9]. Wireless connection system have permitted the majority of the advantages inside our lives, and additionally increased our everyday performance also [2]. Adhoc networks perform without a explained set preserved infrastructure. VANETS dealing with 802.11-structured WLAN advancement today acquired substantial interest. For the reason why that cars constructed with Wi-Fi gear signify the mobile nodes (hosts) [5]. Yet another place by which there is probability of wireless connection system to produce a great impact could be the place of inter-vehicular communications (IVC) [12]. With respect to a wireless system, IVC possess various important aspects: reduced latency due to immediate connection, larger protection and having no service charges [4]. Intervehicle connection (IVC) is developing substantial interest from the research region as well as the vehicle market, by which it'll helpful in offering intelligent transport system
(ITS) along with drivers in addition to tourists relate services [15]. VANETS are the target for manufacturers to wanting to make cars in to intelligent convenience applications [22]. VANETS have caused it to be simpler challenging for layout of numerous safety, comfort and task applications. Collision attentive, street place receptors and readers improvements products the driver crucial information to select the top path in the act to steer clear of the visitors 'rush in addition to incidents. [25]. The particular qualities associated with VANETS enable the introduction of desirable innovative services. The represented applications in the most related areas are safety and comfort to be followed as [3]

1. Comfort Applications: These kinds of program enhance travelers ease and visitor's effectiveness and/or hike the particular path to some location. Some examples for this class contains: visitor detail system, climatic condition details, gas service place or restaurant location and cost details and entertaining connections for example Internet surfing as well as songs download.

2. Safety Applications: The advantages of this class enhance the security of travelers by the way of interchanging security related details through IVC. The details are either given to the driver or utilized to initialize an actuator associated with a dynamic security system.

\subsection{VANET Architecture}

VANET architecture is defined as it is clearly shown in the below figure that the cars are moving in a secured and specified path each of vehicle is defined it paths through the Road Side Unit (RSU) [18,22] through the Security key is defined to each of individual car so that the user can define the correct path. For example, if all the vehicles are running smoothly on the road by well-defined setup of path suddenly a car overtakes the other and the lane changes by default the car got hit to the other car and the crash of two vehicles occurred then the automatic message about this accident will reach the other user through internet and they can control their vehicle direction and speed so that the accident should be avoid and they can change their path easily to get secured lane On Board Unit [5] can help to figure out the problem and it also gives the whole information on board screen of the vehicle which is next to you. The sensors are placed by the road side which gives $\mathrm{u}$ the complete data or information regarding the entrance of new user and the exit of any of the user. 


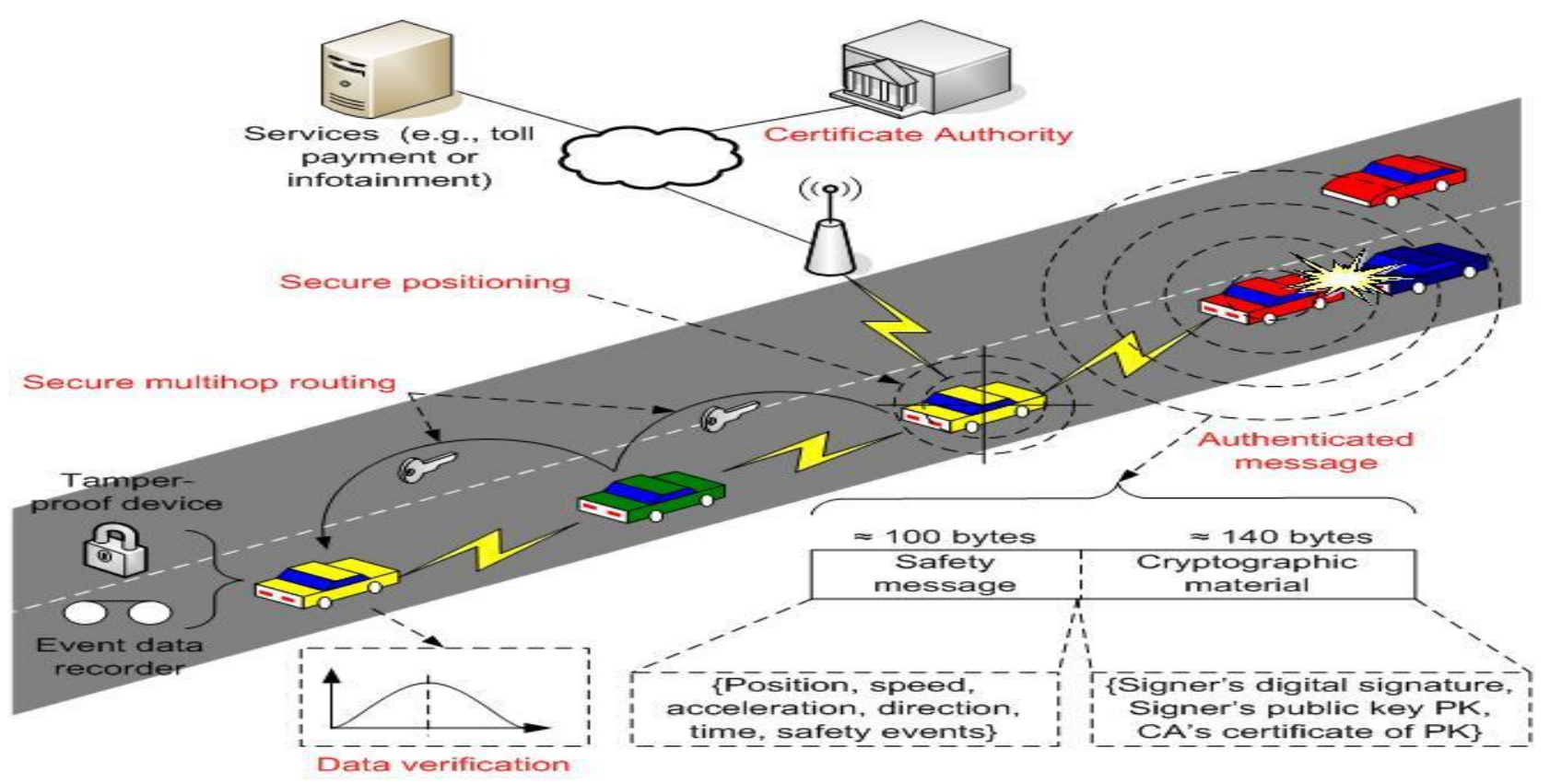

Fig 1.VANET Architecture [24]

\section{TAXONOMY OF ROUTING PROTOCOLS}

Different routing protocols have actually developed for VANETS in many types based on the various factors i.e. divided with several types such as for instance like practices qualities, strategies applied, routing facts, quality of solutions, system structure, routing formulas and so on [39]. Routing algorithms may be divided i.e. based on:

1. Techniques: Topology based, Position based, Geocast protocols, Broadcast protocols, and Cluster-based routing protocols [22].

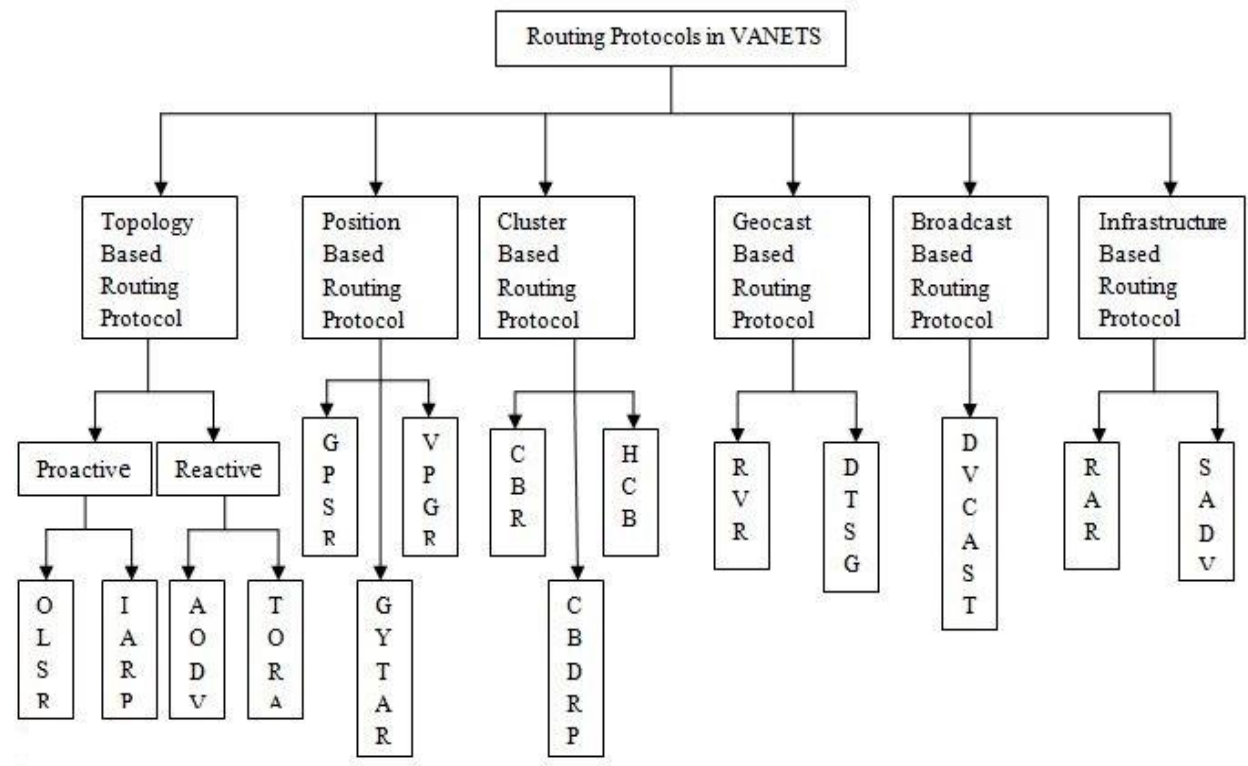

Fig 2: Taxonomy of Routing Protocols in VANETS

\section{ROUTING ATTACKS IN VANETS}

VANET operates on a wireless network, which means security attacks can be triggered by any node in any direction to target any other node in the range. Similarly, other security issues include message replay or delay, message distortion and message leakage. These facts convey the lack of comprehensive defense mechanism in VANET. The VANET needs to have a secure distributed architecture of high mobility nature. The security techniques ideally need to be implemented 'on-demand' and that they should be able to deal with big dynamic clusters at any point in time. There's difficult in handling protection and solitude needs. On a single give, the devices need to produce they can confidence the foundation of information. On another give, the accessibility to such confidence may contradict the solitude needs of the sender. In this kind of assault, the opponents possibly decline the box and interrupt the routing means of the network. Subsequent are the mainly typical routing problems in the VANET. 


\subsection{Black Hole Attack [28]}

A black hole is a region where the network traffic is routed. Nevertheless, often there's number node for the reason this type of region or the nodes stay for the reason that region will not take part in the network. In a dark gap strike, a harmful node presents itself for getting the quickest way to the location node and therefore, tricks the redirecting protocol. As opposed to going for a search on redirecting desk firstly, that hostile node promotes quickly so it includes a new path for the route request. In consequence, opponent node victories the best of responding to the path demand as well as therefore it can intercept the information box or keep it. Once the cast path is properly recognized, this will depend on the harmful node whether to decline or ahead the boxes to wherever it needs determine demonstrates an illustration where in actuality the node A desires to deliver knowledge boxes to node $\mathrm{F}$ but doesn't know the path to F. Thus, A initiates the path finding process. As a harmful node, $\mathrm{N}$ states so it has effective path to $\mathrm{F}$ and pretends so it must certainly be nextnode if A desires to deliver boxes to $\mathrm{F}$.

\subsection{Worm Hole Attack [40]}

In this type an opponent gets packages at one time in the system, tunnels them to some other stage in the system, as well as replays them in to the system from that point. That canal among two opponents are named wormhole. It could be recognized by way of a simple long-range instant or even a sent among the two opponents. Thus it's easy for the opponent to help make the tunneled supply appear prior to different packages given around a standard multi-hop path.

\subsection{Gray Hole Attack [39]}

Here is the expansion of gray hole attack. Such kind of assault the harmful node functions such as the dark node assault nonetheless it lowers the package selectively. It would be performed by three ways.

1. Malicious node may drop incoming packets while allow some packets to pass

2. Malicious node may behave as normal for some time and malicious for a certain time

3. Malicious node may drop incoming packets from some specified nodes for some time and later on it behaves as a normal node. These different types of behavior make attack difficult to detect.

Gray hole attack finally disrupts the network's performance by interfering with the route discovery process.

\subsection{Denial of Service (DOS) Attack}

This type of attack could be carried out by the system insiders as well as outsiders. An insider opponent might be possibly jam the route following shifting fake communications \& ergo, prevents the system link. An outsider opponent may introduction a DOS assault by over and over repeatedly disseminating solid communications with invalid signatures to take the bandwidth and different methods of a under attack vehicle. The affect of the assault is that, VANET failures their power to offer companies to the genuine vehicles. Determine reveals the entire circumstance once the opponent A releases DOS assault in vehicular system and Jams the entire interaction moderate between V2V and V2I. Consequently, reliable customers (B, D, and D) can't speak together in addition to with infrastructure.

\subsection{Illusion Attack [28]:}

In that adversary attempts to deliberately operate his/her warning numbers for providing falsifies details about his/her vehicle. Consequently, the machine effect invokes and fake traffic caution communications are transmitted to neighbors. The influence of the strike is so it can quickly modify the driver's conduct by scattering the incorrect traffic data \& may cause incidents; jams as well as decreases the system effectiveness by losing the bandwidth consumption. Active meaning validation \& meaning strength techniques can not protected systems from this strike since the detrimental car right manipulates \& misleads the detectors of a unique car to make $\&$ transmitted the incorrect traffic information.

\subsection{Sink Hole Attack [29]:}

In Sinkhole attack, a malicious vehicle broadcasts the fake routing data such that it can simply entice most of the system traffic towards it. The affect with this strike is so it makes the system difficult \& degrades the system efficiency often by changing the information boxes or by losing them. Determine demonstrates a Sinkhole strike where a detrimental vehicle lowers the information boxes obtained from the best vehicle $\&$ fake routing data to the reliable vehicles behind it.

\section{RELATED WORK}

Xue yang et al. [1] represented a vehicle-to-vehicle transmission protocol regarding supportive collision warning. One significant specialized concern resolved in that is to attain low-latency in supplying crisis alerts in several street situations. Lars, Wischhof et al. [3] proposed a technique for scalable data dissemination in extremely cellular adhoc systems, it presents method oriented data abstraction and dissemination (SODAD) with this method one application is presented i.e. self-organizing traffic-information system (SOTIS). Tarik, Taleb et al. [7] represents that it decreases the entire traffic in extremely portable VANET networks. The volume of ton needs is paid off by elongating the hyperlink length of the picked paths. The step by step on cars motion data to understand a probable breakage. The system applied behind would be to deliver just unique and well-known boxes named as most readily useful packets. Zhao Zing et al. [9] reveal the many vehicle-assisted knowledge supply (VADD) practices to help you for giving the package towards the best path alongside the tiniest information-delivery delay. Yun Zhou et al. [11] Survey on two well knows algorithms: Ad hoc On-Demand Distance Vector Protocol (AODV) and Optimized Link State Routing Protocol (OLSR), and the performances of AODV and OLSR are analyzed and compared. It is necessary to have an effectual protocol to suit the removal of the main body and to provide possible route for the data transmission. Fethi Filali et al. [14] proposed guideline for the era of vehicular flexibility models. Then, we demonstrate the various techniques opted for by the city for the growth of vehicular flexibility designs and their connections with system simulators. The goal is to supply visitors with a guideline to simply realize and fairly assess the various designs, and ultimately recognize the main one needed because of their demands. Sangman Moh et al. [16] presents a multihop vehicle-to-infrastructure redirecting method called Vertex-Based Predictive Selfish Redirecting (VPGR), which anticipates a string of legitimate vertices (or junctions) from the resource car to set infrastructure (or a roadside unit) in your community of curiosity and, then, forwards knowledge to the set infrastructure through the collection of vertices in metropolitan environments. Bandanjot Kaur et al. [18] discussed the advantages and disadvantages of these routing protocols, it explores the 
motivation behind the designed and trace the evolution of these routing protocols. Wuxiong Zhan et al. [19] produced an logical product with a common radio channel product to completely characterize the accessibility chance and connection chance efficiency in a vehicular relay system considering equally one-hop (direct access) and two-hop (via a relay) communications between an automobile and the infrastructure. Senthil Ganesh N., et al. [23] VANETs might certainly come out to function as marketing program that could help the near future vehicular applications. Writer also analyze the different safety threats and the present methods to overcome the danger facets and display there are productive study initiatives towards creating VANETs a fact in the near future. Swati Verma et al. [28] Security is a major issue in VANET as it can be life threatening. VANET is a subclass of ad hoc network and it is almost same as Mobile Ad Hoc Networks (MANET) but in VANET nodes are vehicles. It is a challenging topic because of frequent link disruptions caused by vehicle mobility. We have used AODV routing protocol in VA NET for proper communication between nodes by forwarding data packets. We have implemented the gray hole attack on routing protocol AODV and shown its impact on implementation of VANET.

\section{COMPARISON TABLE OF ROUTING ATTACKS IN VANETS}

\begin{tabular}{|c|c|c|}
\hline $\begin{array}{l}\text { Routing } \\
\text { Attacks }\end{array}$ & Impact/Effect & $\begin{array}{l}\text { Security } \\
\text { Requirements }\end{array}$ \\
\hline $\begin{array}{l}\text { Denial of } \\
\text { Service } \\
\text { (DOS) Attack }\end{array}$ & $\begin{array}{l}\text { Decreases the } \\
\text { performance \& } \\
\text { efficiency of the } \\
\text { network }\end{array}$ & Availability \\
\hline $\begin{array}{l}\text { Black Hole } \\
\text { Attack }\end{array}$ & $\begin{array}{l}\text { Decreases the } \\
\text { performance \& } \\
\text { efficiency of the } \\
\text { network }\end{array}$ & Availability \\
\hline $\begin{array}{l}\text { Wormhole } \\
\text { Attack }\end{array}$ & $\begin{array}{l}\text { Prevent the discovery of } \\
\text { valid paths as well as } \\
\text { cause information } \\
\text { packets to be lost }\end{array}$ & $\begin{array}{l}\text { Authentication \& } \\
\text { Confidentiality }\end{array}$ \\
\hline $\begin{array}{l}\text { Sinkhole } \\
\text { Attack }\end{array}$ & $\begin{array}{l}\text { Build the network } \\
\text { difficult, either by } \\
\text { modifying the data } \\
\text { packets or by losing } \\
\text { them }\end{array}$ & Availability \\
\hline $\begin{array}{l}\text { Illusion } \\
\text { Attack }\end{array}$ & $\begin{array}{l}\text { Reason vehicles } \\
\text { accidents, traffic jams } \\
\text { as well as decrease the } \\
\text { performance of the } \\
\text { network in terms of } \\
\text { bandwidth consumption }\end{array}$ & Authentication \\
\hline Sybil Attack & $\begin{array}{l}\text { Take over the control of } \\
\text { whole network as well } \\
\text { as inject false } \\
\text { information in it like } \\
\text { traffic congestion, } \\
\text { accident etc }\end{array}$ & Authentication \\
\hline
\end{tabular}

\section{CONCLUSION}

The knowledge of vehicle headway distribution is essential for estimating the probability of connectivity in vehicle ad hoc networks. That report examines numerous redirecting standards of VANET. Developing an effective redirecting method for several VANET purposes is quite hard. Hence study of various VANET standards, researching the different characteristics is totally important to develop new proposals for VANET. Security is an significant matter for routing in VANETs, various applications will effect life-or-death decisions. In this paper represents the study and contrast of various routing attacks in VANETS.

\section{REFERENCES}

[1] X. Yang, J. Liu, F. Zhao, and N. Vaidya, "A vehicle-tovehicle communication protocol for cooperative collision warning," in Proc. Int. Conf, Aug. 2004 pp. 114-123.

[2] J. J. Blum, A. Eskandarian and L. Hoffman, "Challenges of Intervehicle Ad Hoc Networks," IEEE Transactions on Intelligent Transportation Systems, vol. 5, no. 4, pp. 347351, Dec 2004

[3] L. Wischhof, A. Ebner and H. Rohling, "Information Dissemination inSelf- Organizing Intervehicle Networks",IEEE Transaction on Intelligent Transportation Systems, Vol. 6 No. 1, March 2005.

[4] Yousefi, Saleh, Mahmoud Siadat Mousavi, and Mahmood Fathy "Vehicular Adhoc Networks: challenges and perspectives." In ITS Telecommunications Proceedings, $6^{\text {th }}$ International Conference on, pp. 761-766. IEEE, 2006.

[5] Naumov, Valery, Rainer Baumann, and Thomas Gross. "An evaluation of inter- Vehicle Ad hoc Networks based on realistic vehicular traces." In Proceedings of $7^{\text {th }}$ ACM international symposium on Mobile ad hoc networking and computing, pp. 108-119. ACM May 2006.

[6] M. Dorigo, M. Birattari and T. Stutzle, "Ant Colony Optimization: Artificial Ants as A Computational Intelligence Technique," IEEE Computational Intelligence Magazine, vol. 1, no. 4, pp. 28-39, Nov 2006.

[7] Taleb, Tarik, Ehssan Sakhaee, Abbas Jamalipour, Kazuo Hashimoto, Nei Kato and Yoshiaki Nemoto. "A stable routing protocol to support ITS services in VANETS." IEEE Transactions on 56, no. 6 (2007): 3337-3347.

[8] Jerbi, M..Senouci, S.-M. Meraihi and Ghamri-Doudane,Y. (2007), “An improved vehicular adhoc routing protocol for city environments,"Communications 2007. ICC 07 IEEE InternationalConference, pp. 3972-3979, 24-28 June 2007.

[9] Zhao, Jing, and Guohong Cao."VADD: Vehicle-assisted data delivery in vehicular adhoc networks." IEEE transactions on vehicular technology (2008) 1910-1922.

[10] Toor Y, Muhlethaler P, Laouiti A. Vehicle ad hoc networks: applications and related technical issues" Communications Surveys \& Tutorials, IEEE. 2008 Jul 1; 10(3):74-88.

[11] Huawei, Zhan, and Zhou Yun. "Comparison and Analysis AODV and OLSR routing protocols in ad hoc network." In Wireless Communications, Networking and mobile Computing, 2008. WiCOM'08. 4th International Conference on, pp. 1-4. IEEE 2008. 
[12] F. J. Ros, V. Cabrera, J. A. Sanchez, J.A. Martinez and P. M. Ruiz, "Routing in Vehicular Networks techniques, standards and applications eds. H. Moustafa and Y. Zhang, Auerbach Publications, US, April 2009, pp. 109141 .

[13] Liu Y, Bi J, Yang J. Research on vehicular ad hoc networks. InControl and Decision Conference, Chinese (pp. 4430-4435) IEEE Jun 2009.

[14] Härri J, Filali F, Bonnet C. Mobility models for vehicular ad hoc networks: a Survey and taxonomy. Communications Surveys \& Tutorials, IEEE. 2009 Oct $1 ; 11(4): 19-41$

[15] Moustafa, Hassnaa, Sidi Mohammed Senouci, and Moez Jerbi. "Introduction to Vehicular networks." Vehicular Networks (2009).

[16] Shrestha, R.K., Moh, S., Chung, I. and Choi, D" Vertexbased multihop vehicle-to-Infra structure routing for vehicular ad hoc networks".In hics (pp. 1-7).IEEE: (2010)

[17] Wu, Cheng-Shiun, Ai- Pang, and Chih-Shun Hsu. "Design of fast restoration Multipath routing in VANETs." In Computer Symposium International, pp.73-78. IEEE, 2010.

[18] Kohli, Sandhaya, Bandanjot Kaur, and Sabina Bindra. "A comparative study of routing protocols in VANET." Proceedings of ISCET (2010)

[19] S. C. Ng, W. Zhang, Y. Zhang, Y. Yang and G. Mao, "Analysis of Access and Connectivity Probabilities in Vehicular Relay Networks," IEEE Journal on selected areas in Communications, vol. 29, no. 1, pp. 140-150, Jan 2011.

[20] Rondinone, Michele, and Javier Gozalvez. "Exploiting multi-hop connectivity for dynamic routing in VANETs." In Wireless Communication Systems, 8th International Symposium on, pp. 111-115. IEEE, 2011.
[21] Kim JH, Lee S. Reliable routing protocol for vehicular ad hoc networks. AEU International Journal of Electronics and Communications Mar (2011), pp. 68-71.

[22] Mershad, Khaleel, and Hassan Artail. "Performance analysis of routing in VANETS using the RSU network." In Wireless and Mobile Computing, Networking and Communications, IEEE 7th International Conference on, pp. 89-96. IEEE, 2011.

[23] VinhHoa LA, Ana CAVALLI, "Security Attacks And Solutions In Vehicular Ad Hoc Networks: A Survey", International Journal on AdHoc Networking Systems (IJANS) Vol. 4, No. 2, April 2014, pp 1-20.

[24] PriyankaSirola, Amit Joshi, Kamlesh C. Purohit, "An Analytical Study of Routing Attacks in Vehicular Adhoc Networks (VANETs)", International Journal of Computer Science Engineering (IJCSE), Vol. 3 No.04 Jul 2014, pp 210-218.

[25] MeghaNema, Prof. Shalini Stalin, Prof. Vijay Lokhande, "Analysis of Attacks and Challenges in VANET", International Journal of Emerging Technology and Advanced Engineering, Volume 4, Issue 7, July 2014, pp 831-835.

[26] Arif Sari, OnderOnursal, Murat Akkaya, "Review of the Security Issues in Vehicular Ad Hoc Networks (VANET)", Int. J. Communications, Network and System Sciences, 2015, Vol. 8, pp 552-566.

[27] PriyankaSoni , Abhilash Sharma, "Sybil Node Detection and Prevention Approach on Physical Location in VANET ${ }^{e e}$ S", International Journal of Advanced Research in Computer Science and Software Engineering, Volume 5, Issue 7, July 2015, pp 1161-1164.

[28] Swati Verma, BhawnaMallick, PoonamVerma, "Impact of Gray Hole Attack in V ANET", IEEE, 1st International Conference on Next Generation Computing Technologies (NGCT-2015) Dehradun, India, 4-5 September 2015, pp 127-130. 